OPEN ACCESS

Edited by:

John D. Imig,

Medical College of Wisconsin,

United States

Reviewed by:

John M. Seubert,

University of Alberta, Canada

Leonardo Sacconi,

University of Florence, Italy

*Correspondence:

Ruiping Zhao

ruipingzhao@163.com

Jiang Hu

hujiang1961@aliyun.com

${ }^{\dagger}$ These authors have contributed equally to this work.

Specialty section: This article was submitted to

Vascular Physiology,

a section of the journa

Frontiers in Physiology

Received: 13 February 2017 Accepted: 26 May 2017 Published: 13 June 2017

Citation:

Li Y, Meng $X$, Wang W, Liu F, Hao Z, Yang Y, Zhao J, Yin W, Xu L, Zhao $R$ and Hu J (2017) Cardioprotective Effects of SIRT6 in a Mouse Model of Transverse Aortic

Constriction-Induced Heart Failure.

Front. Physiol. 8:394.

doi: 10.3389/fphys.2017.00394

\section{Cardioprotective Effects of SIRT6 in a Mouse Model of Transverse Aortic Constriction-Induced Heart Failure}

\author{
Yongming Li ${ }^{1+}$, Xianda Meng ${ }^{2 \dagger}$, Wenguang Wang ${ }^{1}$, Fu Liu ${ }^{1}$, Zhiru Hao ${ }^{1}$, Yang Yang ${ }^{1}$, \\ Jinbo Zhao ${ }^{1}$, Wensi Yin ${ }^{3}$, Lijuan $\mathrm{Xu}^{3}$, Ruiping $\mathrm{Zhao}^{1,4 *}$ and Jiang $\mathrm{Hu}{ }^{4 *}$ \\ ${ }^{1}$ Department of Cardiology, Baotou Central Hospital, Baotou, China, ${ }^{2}$ Department of Cardiology, Dalian (Municipal) \\ Friendship Hospital, Dalian, China, ${ }^{3}$ Department of Institution of Interventional and Vascular Surgery, Tongji University, \\ Shanghai, China, ${ }^{4}$ Tanslational Medicine Center, Baotou Central Hospital, Baotou, China
}

SIRT6, a member of the NAD (+)-dependent class III deacetylase sirtuin family, plays important roles in the maintenance of cardiovascular homeostasis. Telomere shortening is a risk factor for age-associated diseases, including heart disease. In the present study, we investigated the role of SIRT6 and telomerase in a mouse model of transverse aortic constriction (TAC)-induced heart failure. SIRT6, telomerase reverse transcriptase (TERT), and telomere repeat binding factor (TRF)-1 were significantly downregulated in TAC mice compared with their expression in sham-operated mice. Lentiviral vector-mediated overexpression of SIRT6 upregulated TERT and TRF1 and increased the survival of mice after TAC. Echocardiography and hemodynamic measurements as well as histological analyses indicated that SIRT6 overexpression attenuated TAC-induced heart dysfunction and decreased TAC-induced cardiac inflammatory responses, reducing cardiac fibrosis and decreasing infarct size. Taken together, our findings indicate that SIRT6 protects the myocardium against damage and this effect may be mediated by the modulation of telomeres. Our findings linking SIRT6 and telomere integrity in the heart warrant further investigation into the underlying mechanisms and support SIRT6 as a promising therapeutic target for the treatment of cardiovascular diseases.

Keywords: sirtuin, telomerase reverse transcriptase, telomere repeat binding factor-1, cardiovascular diseases

\section{INTRODUCTION}

Sirtuins or silent information regulator (SIR) proteins are NAD-dependent histone deacetylases that function as regulators of cellular homeostasis through their involvement in energy metabolism, genomic stability, inflammation, oxidative stress, and senescence (Kupis et al., 2016). Seven homologs of yeast Sir2 have been identified, and they differ in subcellular localization, enzymatic activity, and targets. SIRT2 which is present predominantly in the cytoplasm, colocalizes with microtubules and is involved in the deacetylation of the main component of microtubules, $\alpha$-tubulin. SIRT3 is a positive regulator of mitochondrial activity by deacetylating and activating components of the electron transport chain and acetyl-CoA synthetase. SIRT3 is also involved in the defense against oxidative stress. SIRT4, which is located in the mitochondrial matrix, suppresses the secretion of insulin and modulates ATP synthesis. SIRT5 is localized in the mitochondrial matrix, and deacetylates and activates carbamoyl synthetase 1 , which catalyzes the first rate-limiting step of the urea cycle. SIRT7 interacts with and downregulates hypoxia-inducible factors HIF-1 $\alpha$ 
and HIF- $2 \alpha$ and maintains the malignant transformation of cells through H3K18 deacetylation. SIRT7 also regulates mitochondrial homeostasis through GA binding protein $\beta 1$, a regulator of mitochondrial biogenesis and function.

SIRT6, which was recently shown to have a more significant association with increased lifespan than other sirtuins, is a nuclear sirtuin localized to heterochromatin that functions in the deacetylation of many critical genes (Hall et al., 2013). SIRT6 represses the activity of transcription factors involved in aging and inflammation, such as NF- $\kappa \mathrm{B}, \mathrm{c-JUN}$, and HIF- $1 \alpha$ (Zhong and Mostoslavsky, 2010). SIRT6 is highly expressed in endothelial cells, where it confers protection against telomere and genomic DNA damage, thereby preventing the onset of premature senescence (Cardus et al., 2013). SIRT6 prevents cardiac hypertrophy and heart failure, and it contributes to the maintenance of endothelial homeostasis, thus delaying vascular aging (Sundaresan et al., 2012; Kida and Goligorsky, 2016).

Vascular aging involves senescence of endothelial and vascular smooth muscle cells, which can be caused by telomere attrition. Telomeres are chromatin structures that protect the ends of chromosomes from recombination and degradation, thereby preserving DNA integrity and stability (Blackburn, 2001; Blasco, 2005). Telomeric DNA consists of noncoding double stranded repeats of a DNA sequence (TTAGGG in vertebrates), the synthesis of which is catalyzed by telomerase. Telomerase consists of a catalytic subunit (telomerase reverse transcriptase or TERT) and the telomerase RNA component (TERC), which serves as a template for the synthesis of telomeric DNA. Telomeric length and structure are controlled by the binding of telomeric repeat binding factors 1 and 2 (TRF1 and TRF2) to the TTAGGG repeat and the formation of protein complexes. Telomere shortening has been linked to endothelial cell senescence, atherosclerosis, end-stage heart failure, and cardiac hypertrophy among other cardiovascular associated disorders, although whether telomere shortening is a cause or consequence of cardiovascular disease remains unclear (Fuster and Andres, 2006).

In the present study, we investigated the role of SIRT6 and TERT in cardiovascular disease in a mouse model of transverse aortic constriction (TAC)-induced heart failure and explored the underlying mechanisms.

\section{MATERIALS AND METHODS}

\section{Transverse Aortic Constriction (TAC)}

All animal experiments were approved by the medical ethics committee of Central Hospital of Baotou City, and performed in conformance with the Principles of Laboratory Animal Care (National Society for Medical Research) and in accordance with National Institutes of Health guidelines. Mice used in the study were C57BL/6 mice (8-10 weeks of age) purchased from Shanghai Laboratory Animal Center (Shanghai, China) and housed in a facility with a 12-h light/dark cycle at a controlled temperature and humidity with free access to food and water.

The TAC model was generated by tying a suture around the transverse aorta over a 27-gauge blunted needle to cause occlusion of the aorta. After withdrawing the needle, a stenotic aortic lumen was generated. Mice were euthanized by removing the heart under anesthesia. Sham-operated mice without TAC were used as controls.

\section{Lentiviral Construct and Production}

For SIRT6 overexpression, lentiviral vectors were constructed by amplifying the cDNA of SIRT6 by PCR using specific primers (forward primer: $5^{\prime}$-CCGCTCGAGGAAGCGGCCTCA ACAAGG-3', and reverse primer: 5'-CGCGGATCCGTGGTT CCTTCAAGTTCCCC- $3^{\prime}$ ) and subcloning into the pLVX-IRESpuro lentiviral vector using Xho I and BamH I restriction sites (underlined). High titer lentiviruses were generated by transfection of human embryonic kidney 293 cells with recombined vectors. Approximately $48 \mathrm{~h}$ post-transfection, cells were harvested, purified by centrifugation, and stored at $-80^{\circ} \mathrm{C}$ for study.

Mice were injected intravenously via the tail vein with $100 \mu \mathrm{l}$ pLVX-IRES-puro vector (vector) or vector expressing SIRT6 (pSIRT6) lentivirus plasmids (lentivirus at 100 million virus/100 $\mu \mathrm{l}$ PBS/mouse) at 2 weeks and $24 \mathrm{~h}$ before TAC or sham surgery. Myocardial tissue samples were collected at 28 days after surgery. Mice without lentivirus injection served as the control (Ctrl).

\section{Echocardiographic and Hemodynamic Measurements}

Echocardiographic measurements were obtained using a Vevo2100 imaging system (VisualSonics, Toronto, Canada) with a 22-25 MHz linear transducer probe as described previously (Balasubramanian et al., 2015). LV end-diastolic volume (EDV) and end-systolic volume (ESV) were calculated using the Simpson method of disks. Ejection fraction (EF) was determined using the formula $\mathrm{EF}(\%)=(\mathrm{EDV}-\mathrm{ESV}) / \mathrm{EDV} \times 100$. Left ventricular end-diastolic diameter (LVEDD) and end-systolic diameter (LVESD) were measured at one-dimensional mode according to the American Society of Echocardiography guidelines. LV fractional shortening (FS) was calculated with the following formula: FS $(\%)=($ LVEDD - LVESD)/LVEDD $\times$ 100. The maximal rates of rise and fall in $L V$ pressure $(+\mathrm{dP} / \mathrm{dt},-\mathrm{dP} / \mathrm{dt})$ were recorded as previously described (Toldo et al., 2011). The investigator performing and reading the echocardiogram was blinded to the treatment allocation.

\section{Quantitative Real-Time RT-PCR (qRT-PCR)}

For quantitative real-time RT-PCR (qRT-PCR), total RNA was isolated from myocardial tissue samples using the TRIzol reagent (Invitrogen, USA) and reverse transcribed using the SuperScript III kit (Invitrogen) according to the manufacturer's instruction. The PCR primers used were as follows: SIRT6 forward, 5'-CCT GGT CAG CCA GAA CGT AG-3', and reverse, 5'-GTG TCT CTC AGC TCC CCT CT-3'; GAPDH forward, 5' -CCA TCT TCC AGG AGC GAG AC-3', and reverse, 5'-GCC CTT CCA CAA TGC CAA AG-3'. qRT-PCR reactions were performed using SYBR Green reagents (Invitrogen) and on a MyiQ Single-Color Real-Time PCR Detection System (Bio-Rad Laboratories, USA). PCR conditions were $95^{\circ} \mathrm{C}$ for $10 \mathrm{~min}$, then 40 amplification 
cycles of $95^{\circ} \mathrm{C}$ for $10 \mathrm{~s}$ and $72^{\circ} \mathrm{C}$ for $30 \mathrm{~s}$. Reactions were performed in triplicate, and the level of mRNA was normalized to that of GAPDH using the $2^{-\Delta \Delta \mathrm{Ct}}$ method.

\section{Western Blot Analysis}

For western blot analysis, mouse myocardial tissues from infarcted area of TAC groups and anterior left ventricular of sham operated groups were homogenized in radioimmunoprecipitation assay buffer (Beyotime, Shanghai, China) and protein concentration was determined using the BCA protein assay kit (Beyotime). Aliquots containing $20 \mu \mathrm{g}$ of protein were separated by $10 \%$ sodium dodecyl sulfatepolyacrylamide gel electrophoresis and transferred to nitrocelullose membranes (Pall Corporation, USA). Membranes were incubated in 5\% nonfat milk in Tris-buffered saline with Tween 20 (TBST) for $1 \mathrm{~h}$ at room temperature, followed by incubation in primary antibodies at $4{ }^{\circ} \mathrm{C}$ overnight. The primary antibodies used and their dilutions were as follows: anti-SIRT6, 1:2,000; anti- $\alpha$-tubulin, 1:2,000; anti-TERT, 1:1,000; anti-TRF1, $1: 1,000$; anti-collagen I, 1:5,000; anti-FN, 1:1,000; anti- $\alpha$-tubulin, $1: 1,000 ; \beta$-actin, 1:2,000 (all from Abcam, Cambridge, United Kingdom). After washing in TBST, membranes were incubated with the appropriate horseradish peroxidase-conjugated secondary antibodies at room temperature for $1 \mathrm{~h}$. Signal detection was performed using the SuperSignal ECL system (ThermoScientific, Waltham, Massachusetts) and bands were analyzed by ImageJ software. Band intensity was normalized to that of $\alpha$-tubulin or $\beta$-actin.

\section{Assessment of Infarct Size and Survival}

Infarct size was determined using the 2,3,5-triphenyltetrazolium chloride (TTC) staining. Briefly, mice were euthanized 28 days after surgery. The hearts were rapidly removed, frozen, and sliced into transverse sections $(\sim 2 \mathrm{~mm})$. The heart slices were stained with TTC, fixed, and photographed using a digital camera. The infarct area (pale white) in the LV was determined using Meta Morph software (version 6.0, Universal Imaging Corporation). Infarct size was presented as percentage of total LV surface. Survival analysis was performed by daily cage inspection for up to 28 days after surgery.

\section{Histology and Immunohistochemistry}

For histological analyses, mice were euthanized and the hearts were cut into transverse segments, fixed in $10 \%$ formaldehyde, and embedded in paraffin. Sections were cut at a thickness of $10 \mu \mathrm{m}$ and either stained with Masson's trichrome to measure fibrotic areas using ImagePro software. The following antibodies were used for immunohistochemistry analyses: antiLy-6G (1:100, Abcam) to detect neutrophils and anti-CD68 (1:200, Abcam) to detect macrophages. Quantitative assessment of neutrophil and macrophage density was performed by counting the number of Ly6G- and CD68-immunoreactive cells, respectively, in a double-blind fashion from five different fields of the infarcted area at $400 \times$ magnification. For immunofluorescent staining, tissue sections were incubated with anti-SIRT6 antibody (1:100, Abcam) overnight and secondary antibody for $90 \mathrm{~min}$. DAPI solution was then added for $2 \mathrm{~min}$ to stain the nuclei. Images were captured using a Laser-Scanning Confocal Microscope (Olympus FluoView ${ }^{\mathrm{TM}}$ FV1000, Tokyo, Japan).

\section{Enzyme-Linked Immunosorbent Assay (ELISA) Analysis}

TNF- $\alpha$, IL- $1 \beta$, and IL-10 concentrations in mouse hearts were assessed by ELISA using a commercial kit (R\&D System, Minneapolis, MN, USA), according to manufacturer's instruction. Tissues from infarcted area of TAC groups or anterior left ventricular of sham operated group were homogenized in ice-cold PBS buffer containing protease inhibitor cocktail $(21 \mathrm{mmol} / \mathrm{L}$ leupeptin and $3.1 \mathrm{nmol} / \mathrm{L}$ aprotinin), and total proteins were extracted using a protein extraction kit (Pierce, Rockford, IL, USA). Results are expressed as $\mathrm{pg} / \mathrm{mg}$ tissue.

\section{Statistical Analysis}

Data are presented as the mean \pm standard deviation. Data were analyzed with SPSS (version 22.0; SPSS, Chicago, USA) or GraphPad Prism (version 5.0; GraphPad, La Jolla, USA). Student's $t$-test was applied to determine statistically significant differences between groups. One-way analysis of variance followed by Tukey's post-hoc test was applied to determine the significance among groups. $P<0.05$ was considered statistically significant.

\section{RESULTS}

\section{SIRT6 and Telomerase Associated Proteins Are Downregulated in the Myocardium of TAC Mice}

The levels of SIRT6, TERT, and TRF1 were measured in myocardial tissues of mice exposed to TAC or sham surgery to explore the association of sirtuins and telomere function with TAC-induced heart failure. The results of qRT-PCR and western blotting showed that SIRT6 was significantly downregulated in TAC mice at the mRNA and protein levels compared with the levels in sham-operated mice (Figures 1A,B). Immunofluorescence analysis of myocardial tissues confirmed that SIRT6 was downregulated in TAC compared with shamoperated mice (Figure 1C). Western blot analysis showed that TAC significantly downregulated TERT and TRF1 proteins in myocardial tissues compared with their expression in shamoperated mice (Figure 1D).

\section{SIRT6 Overexpression Improves the Survival of TAC Mice and Upregulates Tert and TRF1}

The effect of SIRT6 was further analyzed by injection of a vector expressing SIRT6 lentivirus plasmids into mice before TAC, which resulted in the effective overexpression of SIRT6 mRNA and protein in TAC and sham-operated mice, as indicated by qRT-PCR and western blot analysis (Figures 2A,B). KaplanMeier survival analysis showed that mice overexpressing SIRT6 had significantly better survival rates after TAC than control or 
A

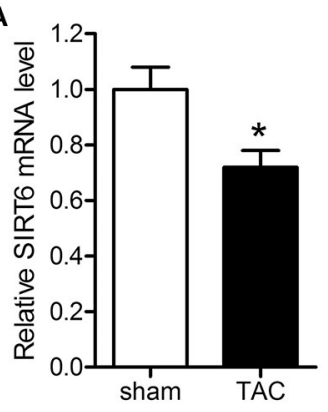

B

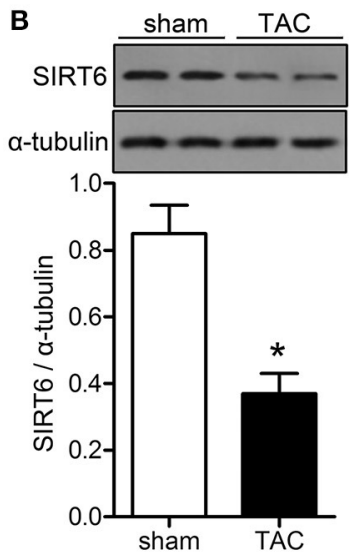

C

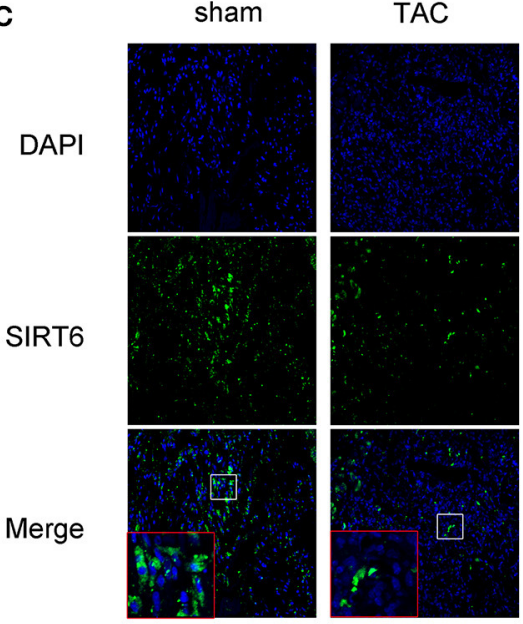

D

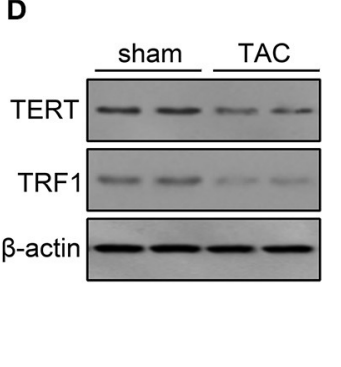

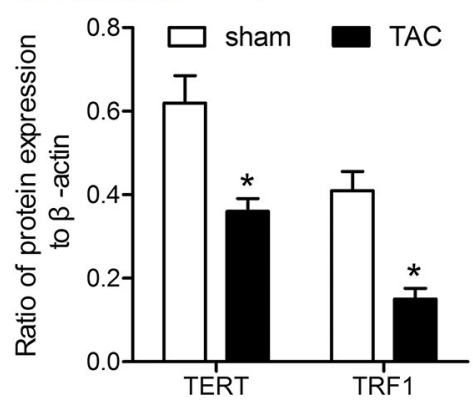

FIGURE 1 | SIRT6 and telomerase associated proteins are downregulated in the myocardium of transverse aortic constriction (TAC) mice. TAC or sham surgery was performed in mice, and myocardial tissue samples were collected at 28 days after surgery. (A) SIRT6 mRNA expression in myocardial tissues was determined by qRT-PCR and normalized to GAPDH expression. ${ }^{*} P<0.05$ vs. sham group. (B) Western blot assessment of the expression levels of SIRT6 normalized to $\alpha$-tubulin expression level. ${ }^{*} P<0.05$ vs. sham group. (C) Immunofluorescence labeling of SIRT6 (green) in myocardial tissues, nuclei were stained with DAPI (blue). $200 \times$ magnification. (D) Western blot assessment of the expression levels of telomere reverse transcriptase (TERT), and telomere repeat binding factor (TRF)1 normalized to $\beta$-actin expression levels. ${ }^{*} P<0.05$ vs. sham group. $n=5$ in the sham group, and $n=8$ in the TAC group.

empty vector-injected mice (Figure 2C). The improved survival occurred in parallel with the upregulation of TERT and TRF1 proteins in TAC mice overexpressing SIRT6 compared with the respective controls (Figure 2D). These results suggested that SIRT6 overexpression protects mice against TAC-induced heart failure by modulating the expression of telomere regulatory proteins.

\section{SIRT6 Overexpression Protects against TAC-Induced Impairment of Cardiovascular Function}

The protective effect of SIRT6 was further analyzed by measuring cardiovascular function after TAC. The results of echocardiographic and hemodynamic measurements showed that SIRT6 overexpression attenuated the effects of TAC on decreasing FS and EF and increasing LVEDD and LVESD (Figures 3A-D). SIRT6 overexpression also attenuated the effects of TAC on the maximal fall and rise of left ventricular pressure compared with that in vector mice (Figures $3 \mathbf{E}, \mathbf{F}$ ). Taken together, these results indicated that SIRT6 exerts a protective effect against TAC-induced cardiovascular function impairment.

\section{SIRT6 Overexpression Decreases TAC-Induced Myocardial Inflammation}

Cardiac inflammation was assessed by measuring neutrophil and macrophage infiltration, which showed that SIRT6 overexpression significantly decreased neutrophil infiltration at 1 day after TAC and attenuated the increase in macrophages at 1 and 7 days after TAC, indicating that SIRT6 decreased cardiac inflammatory responses induced by aortic constriction (Figures 4A-D). In addition, assessment of the levels of TNF$\alpha$, IL- $1 \beta$, and IL-10 by ELISA in the heart homogenate of control and pSIRT6-injected mice after TAC or sham surgery showed that SIRT6 significantly attenuated the TAC-induced upregulation of TNF- $\alpha$ and IL- $1 \beta$ and the downregulation of IL-10 (Figure 4E), suggesting that the effect of SIRT6 was mediated by the modulation of inflammatory factors.

\section{SIRT6 Overexpression Reduces Infarct Size and Cardiac Fibrosis after TAC}

Histological analysis of sections of the heart by Masson's trichrome staining showed that SIRT6 overexpression significantly attenuated the TAC-induced increase in the areas of fibrosis in mice exposed to TAC compared with 


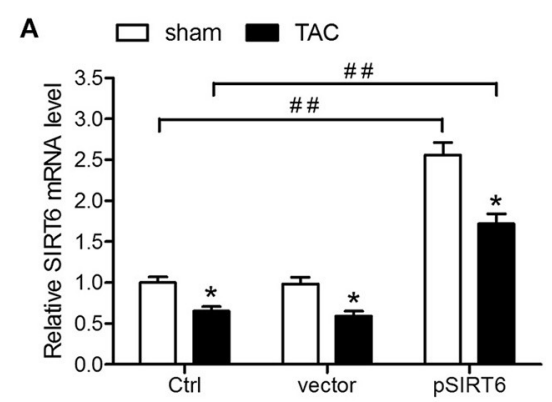

B
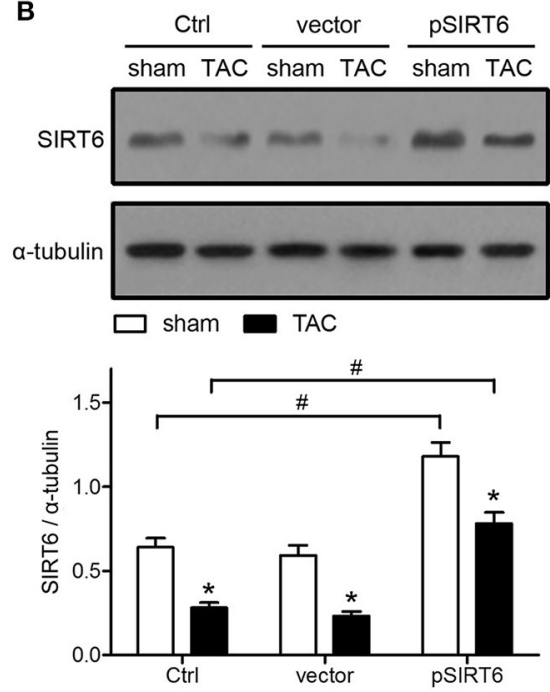

C

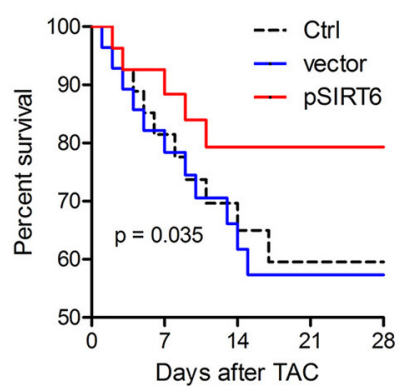

D
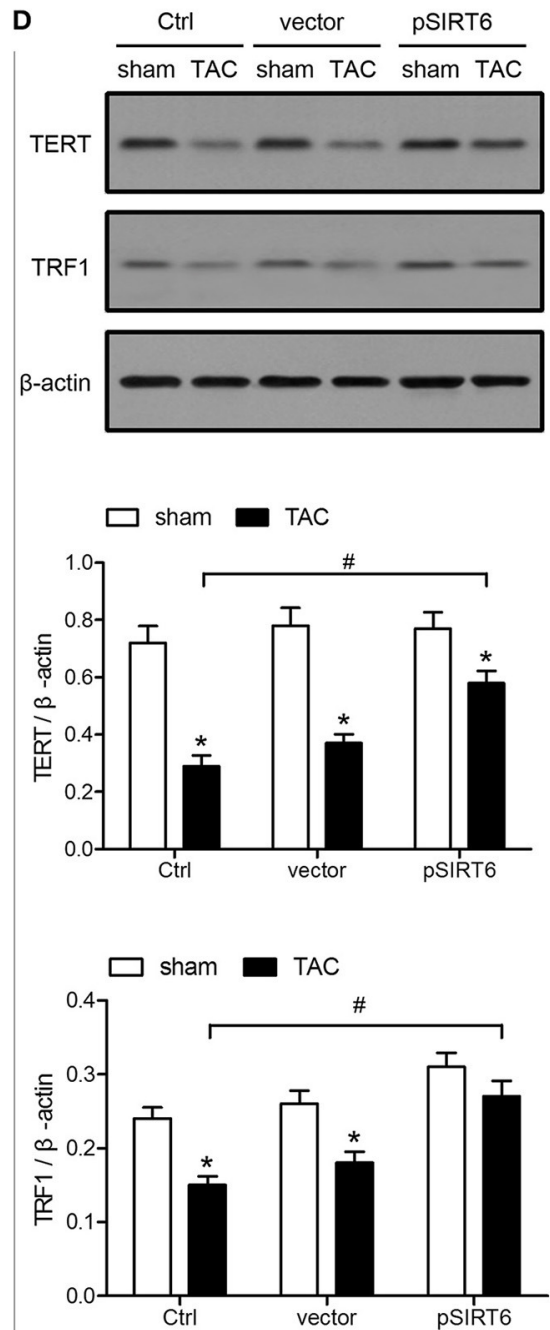

FIGURE 2 | SIRT6 overexpression improves the survival of TAC mice and upregulates TERT and TRF1. Mice were injected intravenously via the tail vein with $100 \mu$ | pLVX-IRES-puro vector (vector) or vector expressing SIRT6 (pSIRT6) lentivirus plasmids at 2 weeks and $24 \mathrm{~h}$ before TAC or sham surgery. Myocardial tissue samples were collected at 28 days after surgery. Mice without lentivirus injection served as the control (Ctrl). (A) SIRT6 mRNA expression in myocardial tissues was determined by qRT-PCR and normalized to GAPDH levels. ${ }^{\star} P<0.05$ vs. sham group, $\# \# P<0.01 . n=8-10$ per group. (B) Protein expression levels of SIRT6 were analyzed by western blotting and normalized to $\alpha$-tubulin expression levels. ${ }^{\star} P<0.05$ vs. sham group, ${ }^{\#} P<0.05$. $n=8-10$ per group. (C) Survival analysis of mice treated as indicated each day after TAC. $n=30$ per group. (D) Western blot assessment of TERT and TRF1 expression normalized to $\beta$-actin levels. ${ }^{\star} P<0.05$ vs. sham group, $\# P<0.05 . n=8$ per group.

sham-operated mice (Figures 5A,B). In addition, heart sections were stained with TTC to determine myocardial infarct size. The results showed that SIRT6 significantly reduced the size of the TAC-induced infarcted area (Figures 5C,D). Western blot analysis showed that SIRT6 significantly attenuated the TAC-induced upregulation of collagen I, fibronectin, and $\alpha$-SMA in myocardial tissues (Figure 5E). Taken together, these results indicated that SIRT6 protects the myocardium against TAC-induced heart failure, improving heart function, reducing infarct size and cardiac fibrosis, and attenuating inflammatory responses, and these effects may be mediated by the modulation of telomere size and structure.

\section{DISCUSSION}

Heart disease is the leading cause of death worldwide, with approximately 17.5 million deaths from cardiovascular disease reported in 2012 according to World Health Organization 

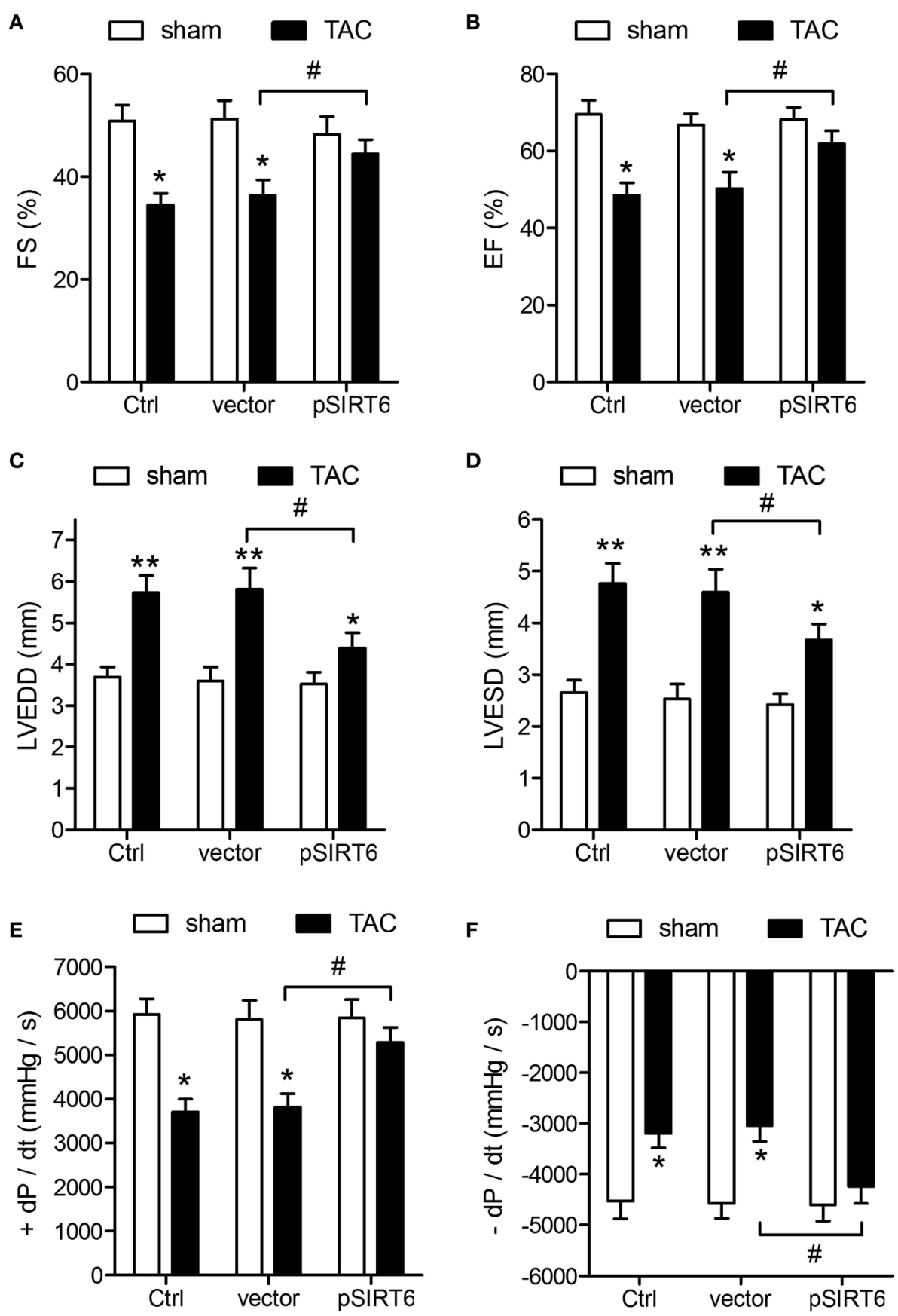

FIGURE 3 | SIRT6 overexpression protects against TAC-induced impairment of cardiovascular function. Echocardiographic and hemodynamic measurements of fractional shortening (FS) (A), ejection fraction (EF) (B), left ventricular end-diastolic dimension (LVEDD) (C), left ventricular end-systolic dimension (LVESD) (D), rates of maximal rise in left ventricular pressure $(+\mathrm{dP} / \mathrm{dt}) \mathbf{( E )}$, and rate of maximal fall in left ventricular pressure $(-\mathrm{dP} / \mathrm{dt})(\mathbf{F})$ in control, vector and pSIRT6-injected mice at 28 days after TAC or sham surgery. ${ }^{\star} P<0.05,{ }^{\star \star} P<0.01$ vs. sham group, ${ }^{\#} P<0.05 . n=12$ per group.

estimates (UnitedHealth Group/National Heart, Lung, and Blood Institute Centres of Excellence, 2014). Extensive research into the molecular mechanisms of cardiovascular disease identified a number of risk factors, and studies suggest that heart failure can be prevented or reverted (Sundaresan et al., 2012). Caloric restriction has been identified as a protective factor with the potential toprevent aging-related cardiovascular diseases (Baur et al., 2012). Sirtuins are mediators of the beneficial effects of caloric restriction, and several sirtuins including SIRT1, SIRT3, and SIRT6 are activated in response to caloric restriction (Cohen et al., 2004; Kanfi et al., 2008; Hirschey et al., 2010). In the present study, we examined the role of SIRT6 

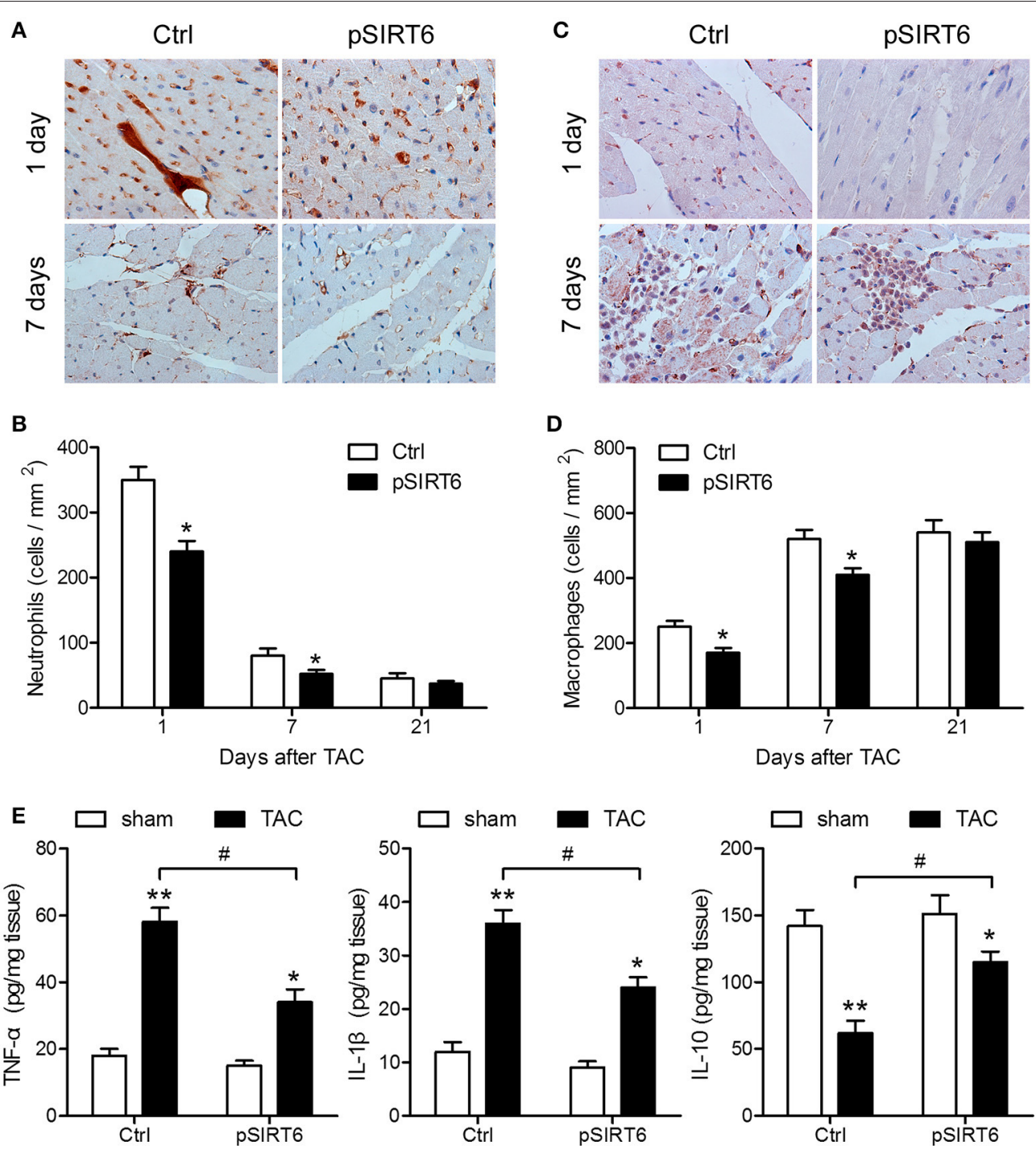

FIGURE 4 | SIRT6 overexpression decreases TAC-induced myocardial inflammation. (A) Representative images of neutrophil (Ly6G + cell) infiltration in control and pSIRT6-injected mice at 1 and 7 days after TAC surgery are shown at $400 \times$ magnification. (B) Quantification of infiltrated neutrophils per area in frozen sections of infarcted hearts at different time points after TAC surgery. (C) Representative images of macrophage (CD68 + cell) infiltration in control and pSIRT6-injected mice at 1 and 7 days after TAC surgery are shown at $400 \times$ magnification. (D) Quantification of infiltrated macrophages per area in frozen sections of infarcted hearts at different time points after TAC surgery. ${ }^{*} P<0.05$ vs. Ctrl group. $n=8$ per group. (E) Levels of TNF- $\alpha, I L-1 \beta$, and IL-10 measured in the heart homogenate of control and pSIRT6-injected mice after TAC or sham surgery for $24 \mathrm{~h} .{ }^{*} P<0.05,{ }^{* *} P<0.01 \mathrm{vs}$. sham group, ${ }^{\#} P<0.05 . n=8$ per group.

in TAC-induced heart failure and the involvement telomere integrity in the protective effects of SIRT6 on cardiovascular function.

The results of the present study showed that SIRT6 was downregulated in mice exposed to TAC compared with its expression in sham-operated mice, and ectopic expression of SIRT6 was associated with increased survival in mice undergoing TAC. Functional and morphological studies showed that SIRT6 overexpression attenuated TAC-induced heart damage and decreased inflammation in the hearts of mice exposed to TAC. Several sirtuins have been shown to exert cardioprotective effects. SIRT1 activates endothelial nitric oxide synthase and protects vascular smooth muscle cells against DNA damage, medial degeneration, and atherosclerosis (Nisoli et al., 2005; Gorenne et al., 2013). SIRT3, a mitochondrial sirtuin, promotes antioxidant defense responses and preserves mitochondrial function, thereby protecting against cardiovascular diseases associated with mitochondrial dysfunction such as dilated cardiomyopathy, heart failure, pulmonary hypertension, and endothelial dysfunction (Winnik et al., 2015). SIRT6, which is highly expressed in the heart, plays a protective role in several cardiovascular-related diseases, including cardiac hypertrophy, heart failure, atherosclerosis, myocardial hypoxic damage, and metabolism (Cai et al., 2012; Maksin-Matveev et al., 2015; Liu et al., 2016). SIRT6 is a negative regulator of the myocardial IGFAkt signaling pathway, the constitutive activation of which leads 
A

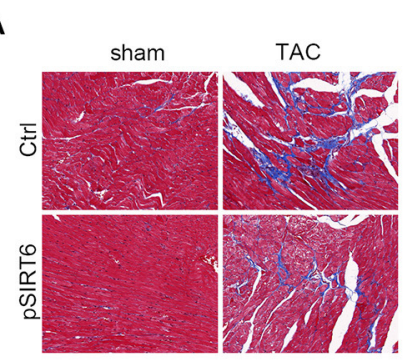

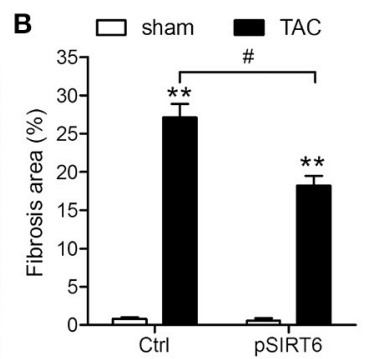
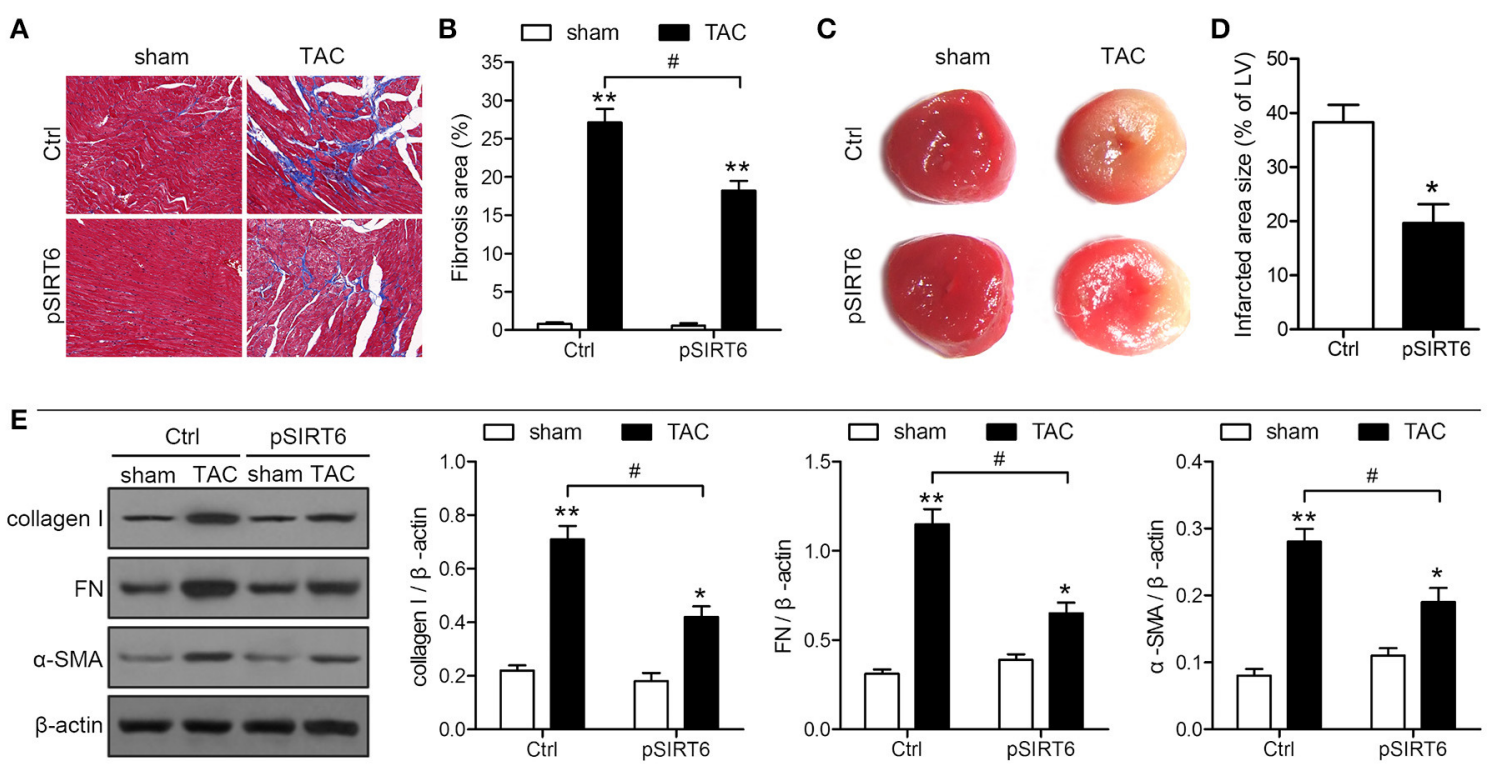

FIGURE 5 | SIRT6 overexpression reduces infarct size and cardiac fibrosis after TAC. Myocardial tissues were collected at 28 days after surgery in control and pSIRT6-injected mice. (A) Representative images of left ventricular sections stained with Masson's trichrome at $200 \times$ magnification. Fibrotic areas are stained in blue. (B) Quantification of fibrotic areas. ${ }^{\star \star} P<0.01$ vs. sham group, $\# P<0.05, n=8$ per group. (C) Representative images of middle heart sections stained with $\Pi$ TC. The infarct area stained pale white. (D) Quantitative analysis of infarct size in TAC mice from TTC stained images. ${ }^{\star} P<0.05$ vs. Ctrl group, $n=6$ per group. (E) Protein expression levels of collagen I, fibronectin (FN) and $\alpha$-SMA were analyzed by western blotting and normalized to $\beta$-actin expression levels. ${ }^{*}<<0.05$, ${ }^{* *} P<0.01$ vs. sham group. ${ }^{\#} P<0.05, n=10$ per group.

to cardiac hypertrophy (Sundaresan et al., 2012). Furthermore, SIRT6 is downregulated in myocardial samples from heart failure patients and in mouse models of cardiac hypertrophy, and SIRT6 overexpression protects the heart from the effects of hypertrophic stimuli, which is consistent with our present findings. SIRT6 acts as a transcriptional repressor of NF- $\kappa$ B, thereby protecting cells against vascular inflammation, a key process in atherogenesis (Libby et al., 2009). SIRT6 associates with telomeres and modulates telomeric chromatin. SIRT6 depletion results in abnormal telomere structure, and studies have shown that the histone deacetylase activity of SIRT6 ensures proper chromatin function, including telomere and genome stabilization, gene expression, and DNA repair, thus preventing cellular senescence (Michishita et al., 2008; Beauharnois et al., 2013).

Telomere length plays an important role in aging related diseases such as atherosclerosis and cardiovascular disease. Telomeres are markers of cellular senescence, and myocardial or vascular dysfunction occurring during aging is related to cellular senescence and loss of function (Matthews et al., 2006; Collerton et al., 2007). Telomeres are involved in the development and progression of cardiovascular diseases even independently of aging, as shown by the presence of shortened telomeres in patients with coronary artery disease or myocardial infarction (Brouilette et al., 2003, 2007). Telomere length in vascular cells affects the development of coronary artery disease by mediating smooth muscle cell and endothelial cell senescence (Ogami et al., 2004; Matthews et al., 2006). Telomere length has also been associated with heart failure, and telomere length is inversely proportional to the severity of heart failure (van der Harst et al., 2007). These studies link the integrity of telomeres to the onset and progression of cardiovascular diseases, although some of the underlying mechanisms remain unclear. In the present study, the downregulation of SIRT6 in TAC mice occurred in parallel with the downregulation of TERT and TRF1, and the increased survival in mice overexpressing SIRT6 was accompanied by the upregulation of these telomereassociated proteins, suggesting that the protective effects of SIRT6 are mediated by the regulation of telomere integrity. However, the exact mechanisms mediating the effect of SIRT6 on cardiac function remain unclear. For example, SIRT6 could affect the structure and function of the heart at the cellular level. Crocini et al. recently described the involvement of Ttubule dysfunction on alterations in $\mathrm{Ca}^{2+}$ release associated with heart failure (Crocini et al., 2014). SIRT1 was suggested to be involved in the modulation of the sarcoplasmic calcium ATPase SERCA2a, which plays an essential role in the maintenance of $\mathrm{Ca}^{2+}$ balance during the cardiac cycle by regulating its transport to the sarcoplasmic reticulum during relaxation (Sulaiman et al., 2010). SERCA2a mRNA is downregulated in patients with dilated or ischemic cardiomyopathy and in aging rat hearts, and downregulation or impaired function of SERCA2a is associated with cardiomyopathy (Tanno et al., 2012). Similarly, SIRT6 could be involved in the regulation of intracellular $\mathrm{Ca}^{2+}$ levels in cardiomyocytes and thus in the maintenance of action potentials in the myocardium. Future studies should focus on exploring the mechanisms by which SIRT6 protects the heart in our in vivo model of TAC-induced heart failure. 
In conclusion, we used a mouse model of TACinduced heart failure to explore the role of SIRT6 and the modulation of telomere length and structure in the protection against cardiovascular dysfunction. We showed that SIRT6 overexpression increased survival, attenuated TAC-induced heart damage and dysfunction, decreased myocardial inflammation induced by TAC, and reduced the areas of fibrosis and infarct size in TAC mice. These effects were accompanied by the upregulation of TERT and TRF1 and the modulation of inflammatory factors, suggesting that SIRT6 protects against heart failure via a mechanism involving the regulation of telomere integrity and inflammatory responses. The ability of sirtuins to improve responses to aging associated stresses makes them potential therapeutic targets, and sirtuin activating compounds have shown promising results in the treatment of inflammatory and metabolic disorders (Bonkowski and Sinclair, 2016). Therefore, our findings are of

\section{REFERENCES}

Balasubramanian, S., Pleasant, D. L., Kasiganesan, H., Quinones, L., Zhang, Y., Sundararaj, K. P., et al. (2015). Dasatinib attenuates pressure overload induced cardiac fibrosis in a murine transverse aortic constriction model. PLoS ONE 10:e0140273. doi: 10.1371/journal.pone.0140273

Baur, J. A., Ungvari, Z., Minor, R. K., Le Couteur, D. G., and de Cabo, R. (2012). Are sirtuins viable targets for improving healthspan and lifespan? Nat. Rev. Drug Discov. 11, 443-461. doi: 10.1038/nrd3738

Beauharnois, J. M., Bolivar, B. E., and Welch, J. T. (2013). Sirtuin 6: a review of biological effects and potential therapeutic properties. Mol. Biosyst. 9, 1789-1806. doi: 10.1039/c3mb00001j

Blackburn, E. H. (2001). Switching and signaling at the telomere. Cell 106, 661-673. doi: 10.1016/S0092-8674(01)00492-5

Blasco, M. A. (2005). Telomeres and human disease: ageing, cancer and beyond. Nat. Rev. Genet. 6, 611-622. doi: 10.1038/nrg1656

Bonkowski, M. S., and Sinclair, D. A. (2016). Slowing ageing by design: the rise of NAD+ and sirtuin-activating compounds. Nat. Rev. Mol. Cell Biol. 17, 679-690. doi: $10.1038 / \mathrm{nrm} .2016 .93$

Brouilette, S. W., Moore, J. S., McMahon, A. D., Thompson, J. R., Ford, I., Shepherd, J., et al. (2007). Telomere length, risk of coronary heart disease, and statin treatment in the West of Scotland Primary Prevention Study: a nested case-control study. Lancet 369, 107-114. doi: 10.1016/S0140-6736(07)60071-3

Brouilette, S., Singh, R. K., Thompson, J. R., Goodall, A. H., and Samani, N. J. (2003). White cell telomere length and risk of premature myocardial infarction. Arterioscler. Thromb. Vasc. Biol. 23, 842-846. doi: 10.1161/01.ATV.0000067426.96344.32

Cai, Y., Yu, S. S., Chen, S. R., Pi, R. B., Gao, S., Li, H., et al. (2012). Nmnat2 protects cardiomyocytes from hypertrophy via activation of SIRT6. FEBS Lett. 586, 866-874. doi: 10.1016/j.febslet.2012.02.014

Cardus, A., Uryga, A. K., Walters, G., and Erusalimsky, J. D. (2013). SIRT6 protects human endothelial cells from DNA damage, telomere dysfunction, and senescence. Cardiovasc. Res. 97, 571-579. doi: 10.1093/cvr/cvs352

Cohen, H. Y., Miller, C., Bitterman, K. J., Wall, N. R., Hekking, B., Kessler, B., et al. (2004). Calorie restriction promotes mammalian cell survival by inducing the SIRT1 deacetylase. Science 305, 390-392. doi: 10.1126/science.1099196

Collerton, J., Martin-Ruiz, C., Kenny, A., Barrass, K., von Zglinicki, T., Kirkwood, T., et al. (2007). Telomere length is associated with left ventricular function in the oldest old: the Newcastle 85+ study. Eur. Heart J. 28, 172-176. doi: 10.1093/eurheartj/ehl437

Crocini, C., Coppini, R., Ferrantini, C., Yan, P., Loew, L. M., Tesi, C., et al. (2014). Defects in T-tubular electrical activity underlie local alterations of calcium release in heart failure. Proc. Natl. Acad. Sci. U.S.A. 111, 15196-15201. doi: $10.1073 /$ pnas. 1411557111 particular importance and merit further investigation into the regulatory role of SIRT6 in the myocardium and the pathways involved.

\section{AUTHOR CONTRIBUTIONS}

$\mathrm{YL}, \mathrm{XM}$, and RZ designed the research, analyzed the data and drafted the manuscript. YL, WW, and FL performed experiments. $\mathrm{ZH}, \mathrm{YY}$, and XM helped for data acquisition and discussion of the data. WY and LX analyzed the data and prepared the figures. JH supervised the whole project. All authors contributed to manuscript writing and edition.

\section{ACKNOWLEDGMENTS}

This work was supported by grants from Baotou Central Hospital.

Fuster, J. J., and Andres, V. (2006). Telomere biology and cardiovascular disease. Circ. Res. 99, 1167-1180. doi: 10.1161/01.RES.0000251281.00845.18

Gorenne, I., Kumar, S., Gray, K., Figg, N., Yu, H., Mercer, J., et al. (2013). Vascular smooth muscle cell sirtuin 1 protects against DNA damage and inhibits atherosclerosis. Circulation 127, 386-396. doi: 10.1161/CIRCULATIONAHA.112.124404

Hall, J. A., Dominy, J. E., Lee, Y., and Puigserver, P. (2013). The sirtuin family's role in aging and age-associated pathologies. J. Clin. Invest. 123, 973-979. doi: 10.1172/JCI64094

Hirschey, M. D., Shimazu, T., Goetzman, E., Jing, E., Schwer, B., Lombard, D. B., et al. (2010). SIRT3 regulates mitochondrial fatty-acid oxidation by reversible enzyme deacetylation. Nature 464, 121-125. doi: 10.1038/nature08778

Kanfi, Y., Shalman, R., Peshti, V., Pilosof, S. N., Gozlan, Y. M., Pearson, K. J., et al. (2008). Regulation of SIRT6 protein levels by nutrient availability. FEBS Lett. 582, 543-548. doi: 10.1016/j.febslet.2008.01.019

Kida, Y., and Goligorsky, M. S. (2016). Sirtuins, cell senescence, and vascular aging. Can. J. Cardiol. 32, 634-641. doi: 10.1016/j.cjca.2015.11.022

Kupis, W., Palyga, J., Tomal, E., and Niewiadomska, E. (2016). The role of sirtuins in cellular homeostasis. J. Physiol. Biochem. 72, 371-380. doi: 10.1007/s13105-016-0492-6

Libby, P., Ridker, P. M., and Hansson, G. K. (2009). Inflammation in atherosclerosis: from pathophysiology to practice. J. Am. Coll. Cardiol. 54, 2129-2138. doi: 10.1016/j.jacc.2009.09.009

Liu, Z., Wang, J., Huang, X., Li, Z., and Liu, P. (2016). Deletion of sirtuin 6 accelerates endothelial dysfunction and atherosclerosis in apolipoprotein E-deficient mice. Transl. Res. 172, 18 e12-29 e12. doi: 10.1016/j.trsl.2016.02.005

Maksin-Matveev, A., Kanfi, Y., Hochhauser, E., Isak, A., Cohen, H. Y., and Shainberg, A. (2015). Sirtuin 6 protects the heart from hypoxic damage. Exp. Cell Res. 330, 81-90. doi: 10.1016/j.yexcr.2014.07.013

Matthews, C., Gorenne, I., Scott, S., Figg, N., Kirkpatrick, P., Ritchie, A., et al. (2006). Vascular smooth muscle cells undergo telomere-based senescence in human atherosclerosis: effects of telomerase and oxidative stress. Circ. Res. 99, 156-164. doi: 10.1161/01.RES.0000233315.38086.bc

Michishita, E., McCord, R. A., Berber, E., Kioi, M., Padilla-Nash, H., Damian, M., et al. (2008). SIRT6 is a histone H3 lysine 9 deacetylase that modulates telomeric chromatin. Nature 452, 492-496. doi: 10.1038/nature06736

Nisoli, E., Tonello, C., Cardile, A., Cozzi, V., Bracale, R., Tedesco, L., et al. (2005). Calorie restriction promotes mitochondrial biogenesis by inducing the expression of eNOS. Science 310, 314-317. doi: 10.1126/science.1117728

Ogami, M., Ikura, Y., Ohsawa, M., Matsuo, T., Kayo, S., Yoshimi, N., et al. (2004). Telomere shortening in human coronary artery diseases. Arterioscler. Thromb. Vasc. Biol. 24, 546-550. doi: 10.1161/01.ATV.0000117200.46938.e7

Sulaiman, M., Matta, M. J., Sundaresan, N. R., Gupta, M. P., Periasamy, M., and Gupta, M. (2010). Resveratrol, an activator of SIRT1, upregulates 
sarcoplasmic calcium ATPase and improves cardiac function in diabetic cardiomyopathy. Am. J. Physiol. Heart Circ. Physiol. 298, H833-H843. doi: 10.1152/ajpheart.00418.2009

Sundaresan, N. R., Vasudevan, P., Zhong, L., Kim, G., Samant, S., Parekh, V., et al. (2012). The sirtuin SIRT6 blocks IGF-Akt signaling and development of cardiac hypertrophy by targeting c-Jun. Nat. Med. 18, 1643-1650. doi: $10.1038 / \mathrm{nm} .2961$

Tanno, M., Kuno, A., Horio, Y., and Miura, T. (2012). Emerging beneficial roles of sirtuins in heart failure. Basic Res. Cardiol. 107:273. doi: 10.1007/s00395-012-0273-5

Toldo, S., Bogaard, H. J., Van Tassell, B. W., Mezzaroma, E., Seropian, I. M., Robati, R., et al. (2011). Right ventricular dysfunction following acute myocardial infarction in the absence of pulmonary hypertension in the mouse. PLoS ONE 6:e18102. doi: 10.1371/journal.pone.0018102

UnitedHealth Group/National Heart, Lung, and Blood Institute Centres of Excellence. (2014). A global research network for non-communicable diseases. Lancet 383, 1446-1447. doi: 10.1016/S0140-6736(13)61808-5

van der Harst, P., van der Steege, G., de Boer, R. A., Voors, A. A., Hall, A. S., Mulder, M. J., et al. (2007). Telomere length of circulating leukocytes is decreased in patients with chronic heart failure. J. Am. Coll. Cardiol. 49, 1459-1464. doi: 10.1016/j.jacc.2007.01.027

Winnik, S., Auwerx, J., Sinclair, D. A., and Matter, C. M. (2015). Protective effects of sirtuins in cardiovascular diseases: from bench to bedside. Eur. Heart J. 36, 3404-3412. doi: 10.1093/eurheartj/ehv290

Zhong, L., and Mostoslavsky, R. (2010). SIRT6: a master epigenetic gatekeeper of glucose metabolism. Transcription 1, 17-21. doi: 10.4161/trns.1.1.12143

Conflict of Interest Statement: The authors declare that the research was conducted in the absence of any commercial or financial relationships that could be construed as a potential conflict of interest.

Copyright (c) 2017 Li, Meng, Wang, Liu, Hao, Yang, Zhao, Yin, Xu, Zhao and Hu. This is an open-access article distributed under the terms of the Creative Commons Attribution License (CC BY). The use, distribution or reproduction in other forums is permitted, provided the original author(s) or licensor are credited and that the original publication in this journal is cited, in accordance with accepted academic practice. No use, distribution or reproduction is permitted which does not comply with these terms. 